\title{
Building Biomechanical Models Based on Medical Image Data: An Assessment of Model Accuracy
}

\author{
Wendy M. Murray ${ }^{1}$, Allison S. Arnold ${ }^{2}$, Silvia Salinas ${ }^{2}$, \\ Mahidhar M. Durbhakula ${ }^{2}$, Thomas S. Buchanan ${ }^{3}$, and Scott L. Delp ${ }^{2}$ \\ ${ }^{1}$ Biomedical Engineering Department, Case Western Reserve University \& \\ Rehabilitation Engineering Center, MetroHealth Medical Center, 2500 MetroHealth Dr., \\ Cleveland, OH 44109-1998, U.S.A. \\ wmm@po.cwru.edu \\ ${ }^{2}$ Departments of Biomedical Engineering and Physical Medicine \& Rehabilitation, \\ Northwestern University \& Sensory Motor Performance Program, Rehabilitation \\ Institute of Chicago, Room 1406, 345 East Superior St., Chicago, IL 60611, U.S.A \\ \{asarnold, s-salinas, m-durbhakula, s-delp\}@nwu.edu \\ ${ }^{3}$ Mechanical Engineering Department, University of Delaware, Newark, DE 19716, \\ U.S.A. \\ buchanan@me.udel.edu
}

\begin{abstract}
The goals of this work were to (i) establish a method for building subject-specific biomechanical models from medical image data, (ii) construct a subject-specific model of the elbow, and (iii) quantify the accuracy of soft tissue excursions estimated from the model. We developed a kinematic model of the elbow joint and its surrounding musculature from magnetic resonance images of a 64" male cadaver specimen in one limb position. Moment arms estimated from the model (i.e., the changes in muscle-tendon lengths with elbow flexion angle) were compared to moment arms measured experimentally from the same specimen. In five of the six muscles studied, the model explained $84 \%-94 \%$ of the variation in the experimental data. Model estimates of peak elbow flexion moment arm were within $13 \%$ of the experimental peaks. Our results suggest that subjectspecific musculoskeletal models derived from medical image data have the potential to substantially improve estimates of soft tissue excursions in living subjects.
\end{abstract}

\section{Introduction}

Quantification of soft tissue excursions that occur during movement is important for the planning of orthopaedic surgical interventions, such as tendon transfers and osteotomies. Surgeons frequently introduce changes in muscle force- and momentgenerating properties by modifying the length and moment arm of a muscle. Although these changes are usually intended to improve function (e.g., tendon transfer surgeries 
which enhance hand grasp function in persons with spinal cord injury), they can leave patients with weak or dysfunctional limbs if the muscle fibers are too long or too short postoperatively to generate active force or if the moment arms of the muscles are compromised. Predicting the biomechanical consequences of surgical alterations, therefore, requires detailed knowledge of the soft tissue lengths and excursions before and after the intervention.

Muscle-tendon lengths and moment arms are commonly estimated in cadaver specimens (e.g., $[1,2]$ ) or using nominal biomechanical models (e.g., [3, 4]). However, it remains unclear whether "averaged" experimental data or generic models accurately represent populations that have not been studied in anatomical preparations, such as children or individuals with pathologies (e.g., bone deformities or muscle contracture). Because patients undergoing surgical reconstructions of musculoskeletal structures frequently fall into this category, methods that enable soft tissue excursions of individual subjects to be estimated in vivo are needed.

Computer-generated three-dimensional (3D) reconstructions from computed tomography (CT) or magnetic resonance (MR) images provide an accurate, noninvasive method to quantify musculoskeletal anatomy in living subjects $[5,6]$. However, accurate measurements of muscle-tendon excursions from medical image data would require extensive imaging protocols to capture the orientations and positions of the muscles in several different joint positions. The combination of medical imaging and graphics-based musculoskeletal modeling is a promising alternative for estimating muscle-tendon lengths and moment arms in living subjects. For instance, a 3D reconstruction of a limb in one joint position can be animated by making basic assumptions about joint kinematics and musculotendon travel with joint rotation. This hybrid method would drastically reduce the time, expense, and data processing concerns that currently limit the applications of 3D reconstructions of medical image data. However, methods for creating subject-specific biomechanical models need to be established. Also, it is critical to demonstrate the accuracy of any model before it can be used to guide patient-specific treatment decisions.

The specific aim of this study was to create a biomechanical model of the elbow joint and its surrounding musculature from MR images and to quantify the accuracy of muscle moment arms estimated using the model. Because a muscle's moment arm determines its change in length over a range of motion [7], our assessment of moment arms in this study also provides a rigorous test of our model's capacity to estimate muscle-tendon lengths and excursions.

\section{Methods}

A 3D reconstruction of the upper extremity harvested from a 6'4" male cadaver specimen was created from MR images, and a kinematic model of the elbow joint and its surrounding musculature was constructed based on the 3D surface models of the muscles and bones. To determine the accuracy of the model over a range of elbow flexion, moment arms of the brachioradialis, biceps, extensor carpi radialis longus (ECRL), brachialis, pronator teres, and triceps estimated from the model were 
compared to the moment arms determined experimentally from the same specimen. We also compared the moment arms of the 6'4" specimen to the moment arms estimated from a $5^{\prime} 0^{\prime \prime}$ specimen to quantify differences that might be observed between individuals of different sizes and to provide some context for evaluating the errors of our model.

\subsection{Construction of the 3D Surfaces}

Axial and sagittal images of the upper extremity specimen were obtained using a GE Signa MRI Scanner (Series 2, 1.5T). Five overlapping series of T1-weighted spinecho images were collected (Table I). In four of the image series, the specimen was scanned in the transverse plane from the proximal scapula to the wrist joint with the elbow fully extended and the forearm pronated approximately $60^{\circ}$. In the fifth series, sagittal images of the elbow joint were obtained with the elbow flexed approximately $30^{\circ}$ and the forearm pronated $60^{\circ}$. All series were scanned in contiguous $3 \mathrm{~mm}$ slices using an extremity coil, except for the proximal humerus which was scanned using a license plate surface coil. To facilitate registration of the images, multiple Vitamin E capsules were secured to the specimen at the axilla of the upper arm, the distal humerus, and the proximal forearm.

Table 1. MR Imaging Protocols

\begin{tabular}{|c|c|c|c|c|c|}
\hline & $\begin{array}{c}\text { DISTAL } \\
\text { FOREARM }\end{array}$ & $\begin{array}{c}\text { ELBOW } \\
\text { JoINT } \\
\text { (AXIAL) } \\
\end{array}$ & $\begin{array}{c}\text { MID- } \\
\text { HuMERUS }\end{array}$ & $\begin{array}{c}\text { Proximal } \\
\text { Humerus }\end{array}$ & $\begin{array}{c}\text { ELBOW } \\
\text { JoINT } \\
\text { (SAGITTAL) }\end{array}$ \\
\hline $\begin{array}{c}\text { Pulse } \\
\text { Sequence } \\
(m s)\end{array}$ & $\begin{array}{c}\mathrm{TR}=316 \\
\mathrm{TE}=16\end{array}$ & $\begin{array}{c}T R=400 \\
T E=16\end{array}$ & $\begin{array}{c}\mathrm{TR}=400 \\
\mathrm{TE}=16\end{array}$ & $\begin{array}{c}\mathrm{TR}=500 \\
\mathrm{TE}=16\end{array}$ & $\begin{array}{c}\mathrm{TR}=300 \\
\mathrm{TE}=15\end{array}$ \\
\hline Matrix & $256 \times 128$ & $256 \times 128$ & $256 \times 128$ & $256 \times 128$ & $256 \times 256$ \\
\hline FOV $(\mathrm{cm})$ & $28 \times 28$ & $28 \times 28$ & $28 \times 28$ & $40 \times 40$ & $20 \times 20$ \\
\hline NEX & 1.5 & 1.5 & 1.5 & 1.5 & 2 \\
\hline \# Slices & 72 & 63 & 65 & 87 & 35 \\
\hline
\end{tabular}

Boundaries of the humerus, radius, ulna, the long head of the biceps, the brachialis, the brachioradialis, the ECRL, the pronator teres, and the lateral head of the triceps were identified manually in individual slices. A 3D surface representing each anatomical structure was created from the two-dimensional boundaries in serial images. Muscle attachment sites on the bones were identified by placing markers within the images. Three-dimensional surface models of the Vitamin $\mathrm{E}$ capsules visible in each series were also created. The homogeneous transformations between 
overlapping series of images were calculated from the centroids of the Vitamin $\mathrm{E}$ capsules using a least-squares algorithm [8].

\subsection{Development of the Kinematic Model}

The bone and muscle surfaces obtained from the MR images were used to construct a kinematic model of the elbow joint and its surrounding musculature (Fig. 1). Elbow flexion-extension was modeled as a uniaxial hinge joint with its axis passing through the centers of the capitulum and trochlear groove $[9,10,11,12]$. The center of the capitulum was estimated from a sphere that was fit to its surface. Similarly, the center of the trochlear groove was estimated by fitting a circle to the trochlear groove. Elbow flexion was defined from full extension $\left(0^{\circ}\right.$ flexion) to $130^{\circ}$ flexion.

The lines of action of the elbow muscles were represented in the model as a series of points connected by line segments. Origin and insertion points were defined based on the centroids of the muscle attachment sites on the bones. Due to the curvature of the humeral shaft, the calculated centroid of the brachialis origin did not lie on the bone surface. This point was translated anteriorly to place the origin on the surface of the humerus. Because the scapula was not imaged, an effective origin for the biceps was defined along the intertubercular sulcus. Similarly, because the hand was not imaged, an effective origin of ECRL was defined on the distal radius.

In addition to origin and insertion points, intermediate 'wrapping' points were defined as a function of elbow flexion angle. The locations of the wrapping points in full extension were based on the 3D muscle surfaces. As the elbow was flexed, wrapping points that were a shorter distance from the axis of rotation than both of a muscle's attachment sites were removed from the path because a recent anatomical study has shown that the shortest distance between the flexion-extension axis and an elbow muscle's attachment sites indicates peak moment arm [13]. For the triceps, wrapping points were added to the path during flexion to model the tendon of the triceps wrapping over the olecranon process of the ulna. We constrained the models of the muscle-tendon paths so that introducing or removing an intermediate point did not cause an instantaneous change in moment arm or muscle-tendon length. Intermediate 'via' points were also defined, and remained in the model over the entire range of motion to prevent the paths from penetrating bone surfaces. The via points in the model had no substantial effects on the estimated moment arms. Moment arms were estimated with the model using the partial velocity method [14].

\subsection{Determination of Muscle Moment Arms from Tendon Displacement Data}

Elbow flexion moment arms were estimated experimentally in both the 64" male specimen and a $5^{\prime} 0$ " female specimen. Using the tendon displacement method [7], moment arm $(m a)$ was calculated as the partial derivative of muscle-tendon length $(\partial \ell)$ with respect to elbow flexion angle $(\theta)$. That is, 


$$
m a=\frac{\partial \ell}{\partial \theta}
$$

The shoulder joint was disarticulated, the skin and fascia proximal to the wrist joint were removed, and the muscles were prepared as described previously [2]. The upper extremity was mounted on a horizontal surface, supported at the humeral head and the medial epicondyle. Elbow flexion angle was measured with an electrogoniometer (Penny and Giles Biometrics, United Kingdom). Tendon displacement measurements were made by connecting each muscle to a Celesco PT101 position transducer (Celesco Transducer Products, Canoga Park, CA) with a wire and slowly moving the forearm through approximately $120^{\circ}$ of elbow flexion. The forearm was maintained in neutral $\left(0^{\circ}\right.$ pronation/supination) during moment arm measurements.

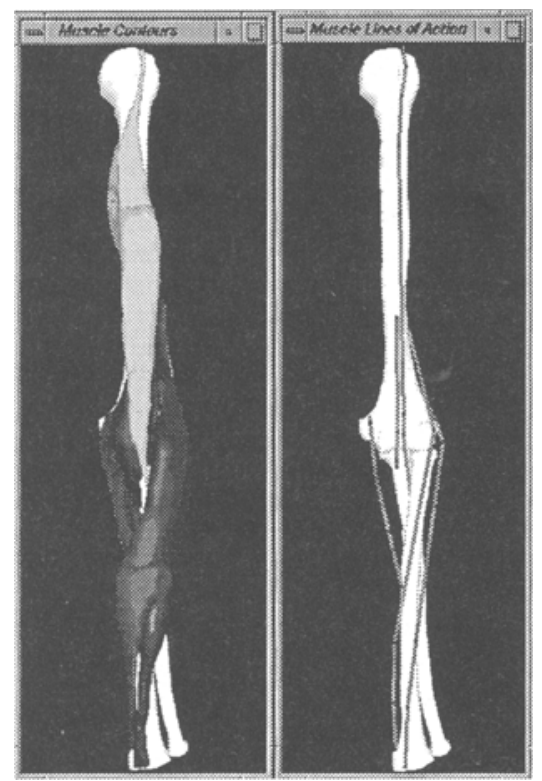

Fig. 1. Computer graphics reconstruction on the $64^{\prime \prime}$ male specimen (left), illustrating the 3D muscle and bone surfaces, and the kinematic model of the elbow joint (right), where muscle lines of action were represented as a series of points connected by line segments

The outputs of the position transducer and the electrogoniometer were sampled at $15 \mathrm{~Hz}$. Five trials of tendon displacement vs elbow flexion angle were collected per muscle. The numerical derivative of each trial was digitally filtered using a second order Butterworth filter with a cut-off frequency of one radian ${ }^{-1}$. The five filtered derivatives were averaged to estimate moment arm. Moment arms were estimated between $20^{\circ}-120^{\circ}$ flexion for the elbow flexors and $30^{\circ}-120^{\circ}$ flexion for triceps. 


\subsection{Assessment of the Accuracy of the Model}

The moment arm vs elbow flexion angle curves estimated using the MR based model were quantitatively compared to the moment arms determined experimentally for the large male specimen. For each muscle, the correlation coefficient and the root mean square (RMS) error between the model estimate and the anatomical data were calculated. The square of the correlation coefficient denotes the variation in the experimental data that is accounted for by the model. The RMS error is a measure of the average, absolute value of the difference between the model estimate and the experimental data. That is,

$$
R M S=\sqrt{\frac{1}{N} \sum_{i=1}^{N}\left(m a_{i}^{D}-m a_{i}^{M}\right)^{2}}
$$

where $\mathrm{N}$ is the number of data points, $m a_{i}^{D}$ is the $\mathrm{i}^{\text {th }}$ value of the experimental data, and $m a_{i}^{M}$ is the corresponding value of the model moment arm. We also compared the model estimate of peak moment arm to the peak from the experimental data. Quantitative comparisons between the experimental data from the 6 64 " and $5{ }^{\prime} 0$ " cadaver specimens provided a means to evaluate the significance of the model errors.

\section{Results}

The MR based model captured the salient features of the experimental data. In five of the six muscles studied, the correlation coefficients between the model curves and the experimentally determined moment arms were greater than 0.9 , and the model explained $84 \%-94 \%$ of the variation in the data (Fig. 2). RMS errors of the model ranged from $3 \mathrm{~mm}$ to $7 \mathrm{~mm}$ (Fig. 3), and the model estimates of peak elbow flexion moment arms were within $13 \%$ of the peaks estimated experimentally (Fig. 4).

Errors in the moment arms from the MR model were generally smaller than differences between the 6 6 $4^{\prime \prime}$ male specimen and the $5^{\prime} 0^{\prime \prime}$ female specimen. For instance, the errors in the model estimates of peak moment arms were substantially smaller than the differences between peaks in the large and small specimens (Fig. 4). However, for four muscles (biceps, brachialis, pronator teres, and triceps), the model RMS errors were comparable to the differences between the experimental data sets (Fig. 3). For pronator teres and triceps, this occurred because there were only small differences between the moment arms from the $5^{\prime} 0^{\prime \prime}$ female and the $64^{\prime \prime}$ male (Fig. 5 , compare differences in PT and TRI curves to differences in BRD curves). In contrast, the model RMS errors for biceps and brachialis were relatively large because the model estimates were shifted versions of the experimental curves (Fig. 2B). If the model estimates of biceps and brachialis moment arms were shifted by $-20^{\circ}$, the model's RMS errors would decrease to $2 \mathrm{~mm}$ and $4 \mathrm{~mm}$, respectively. Similarly, given a $-20^{\circ}$ angular shift, the correlation coefficients would increase from 0.916 to 0.998 (biceps) and from 0.783 to 0.987 (brachialis). Thus, the model estimates of biceps and brachialis moment arms could be significantly improved if the source of the angular 
discrepancy between the model and the experimental data could be identified and addressed.

Of the six muscles evaluated, the brachialis model yielded the least accurate estimate of muscle moment arm. Unlike the other muscles, peak moment arm was not estimated to within $10 \%$ by the model, and the model explained only $61 \%$ of the variation in the brachialis experimental data. Brachialis has a broad origin on the humerus, and the discrepancy between model and experimental curves may be due to limitations in (i) representing muscles with broad attachments with single origin or insertion points or (ii) obtaining accurate experimental estimates of moment arms from muscles with broad attachment sites.
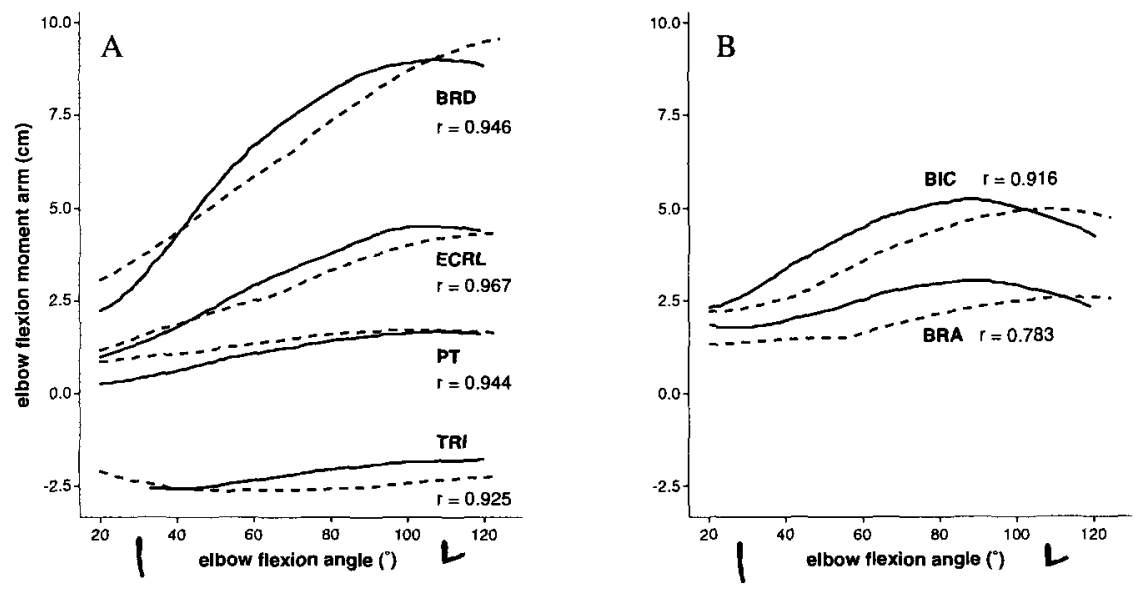

Fig 2. (A). Elbow flexion moment arms of brachioradialis (BRD), ECRL, pronator teres (PT), and triceps (TRI) estimated with the MR based model (dashed lines) and estimated using the tendon displacement method in the same cadaver specimen (solid lines). (B). Elbow flexion moment arms of biceps (BIC) and brachialis (BRA) estimated with the MR based model (dashed lines) and estimated using the tendon displacement method (solid lines). With the exception of the brachialis, the correlation coefficients ( $r$ ) between the model estimates and the anatomical data were greater than 0.9

\section{Discussion}

Orthopaedic surgical procedures frequently alter the lengths and moment arms of muscles; however, they are planned with little quantitative data that describe muscle function before surgery, or how muscle function might be altered postoperatively. While generic musculoskeletal models have provided some useful clinical insights (e.g., [15]), the accuracy and reliability of generic models for estimating soft-tissue excursions in individuals of different sizes, ages, and pathologies has not been tested. 
We believe the development of accurate, time-effective and cost-effective methods to build subject-specific biomechanical models from medical images could provide important insights needed to design and implement more effective surgical procedures.

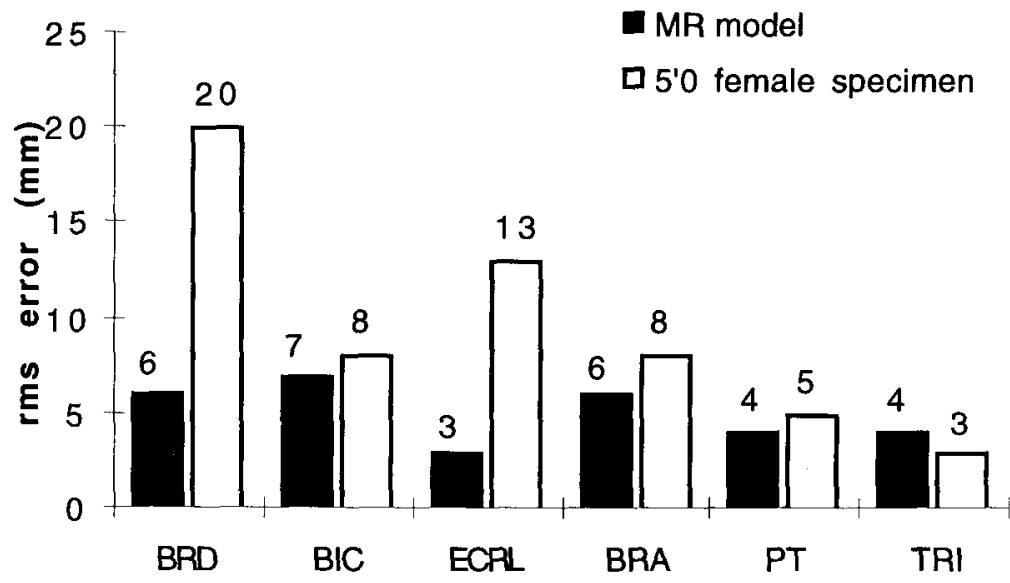

Fig. 3. RMS errors for the MR based model (filled bars) and the $5^{\prime} 0^{\prime \prime}$ female specimen (white bars) for brachioradialis (BRD), biceps (BIC), ECRL, brachialis (BRA), pronator teres (PT), and triceps (TRI). The RMS error for the model ranged from $3 \mathrm{~mm}$ (ECRL) to 7 $\mathrm{mm}$ (BIC). For BRD and ECRL, the RMS error of the model was substantially smaller than the error from the data from the $5^{\prime}{ }^{\prime \prime}$ "female specimen

In this study, we created a "specimen-specific" kinematic model of the elbow joint and its surrounding musculature, and we quantified the accuracy of muscle moment arms estimated using the model. The model was developed from a minimal set of medical image data - a 3D reconstruction of an upper extremity specimen in a single joint position. Nevertheless, the model was able to capture the salient features of elbow flexion moment arms determined experimentally on the same specimen. Clearly, previous studies which characterized elbow joint kinematics $[9,10,11,12]$ and identified the determinants of peak elbow flexion moment arm [13] guided the development of our model and contributed to the success of our approach. It may be more difficult to develop accurate models of joints with more complex kinematics and muscle-tendon paths, such as the shoulder or the knee, or to characterize joint kinematics a priori in a patient with bone deformities. Not surprisingly, the largest source of error in our moment arm estimates was the variation in the moment arms with elbow flexion angle. Further investigation of how muscle-tendon paths change with joint motions in vivo would improve the reliability of biomechanical models constructed from a minimal set of medical images.

The results of our study suggest that subject-specific models could greatly improve estimates of soft-tissue excursions in living subjects. Few investigators have attempted to quantify differences in moment arms across differently sized specimens, and most existing musculoskeletal models represent an adult male of average stature. 


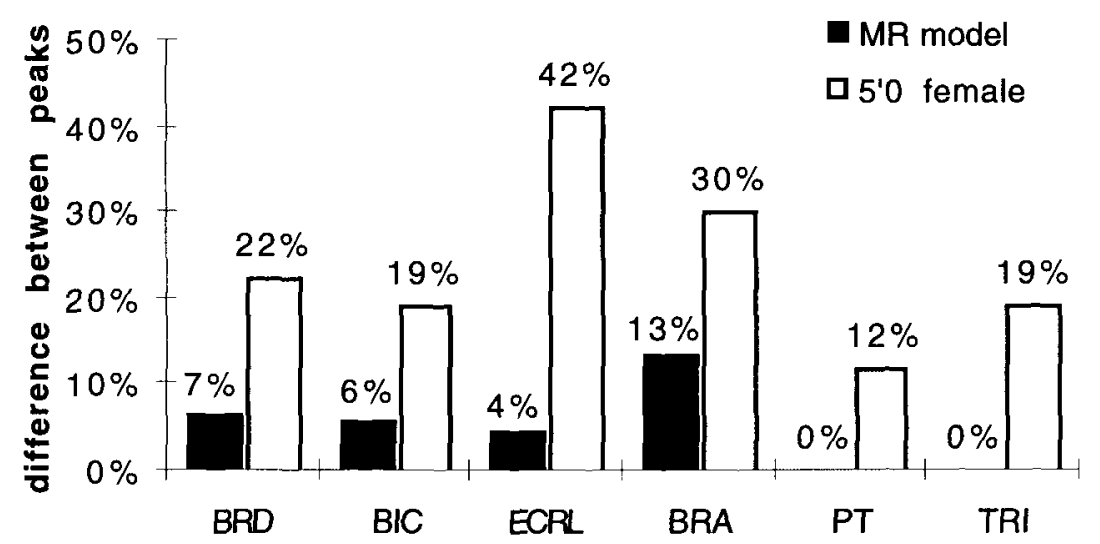

Fig 4. Differences between peak moment arms estimated with the MR based model and determined experimentally from the 6'4" male specimen (filled bars) and differences between the peak moment arms of the two cadaver specimens (white bars). Differences between peaks are expressed as a percentage of the experimentally determined peak from the male specimen. For each muscle, the MR based model provided a more accurate estimate of peak moment arm compared to the specimen of different anthropometric dimensions
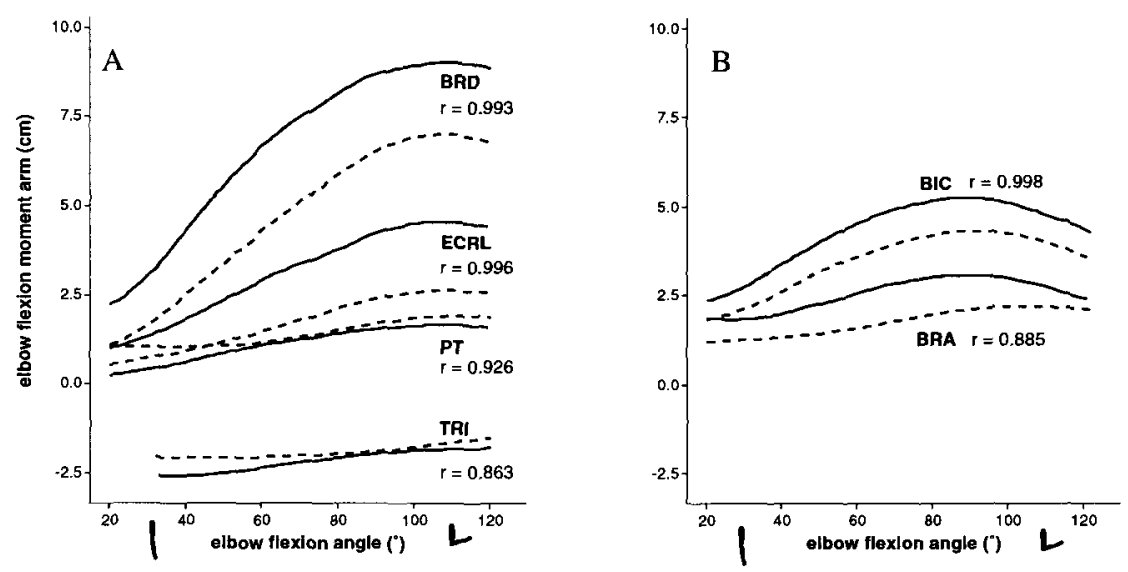

Fig 5. (A). Elbow flexion moment arms of brachioradialis (BRD), ECRL, pronator teres (PT), and triceps (TRI) determined experimentally from the $5^{\prime} 0^{\prime \prime}$ female specimen (dashed lines) and from the 6'4" male specimen (solid lines). (B). Elbow flexion moment arms of biceps (BIC) and brachialis (BRA) determined experimentally from the $5^{\prime} 0^{\prime \prime}$ female specimen (dashed lines) and from the 6'4" male specimen (solid lines). There are substantial differences in moment arm magnitudes between the two specimens for BRD, ECRL, BIC, and BRA, but not for the PT and TRI 
In this study, we found that errors in the elbow flexion moment arms estimated from the MR based model were generally smaller than differences between the $6^{\prime} 4^{\prime \prime}$ male specimen and a $5^{\prime} 0^{\prime \prime}$ female specimen. The moment arms of the most commonly referenced experimental data [16] are similar to the moment arms of the 5'0" specimen in our study, indicating that the MR based model provides a more accurate representation of the $64 "$ specimen than the generally accepted literature standard. Peak moment arms estimated using a commonly cited kinematic model of the elbow joint [17] are comparable to the MR based estimates for some muscles (biceps, brachialis, triceps), but are substantially smaller for others (brachioradialis, ECRL, pronator teres). For individuals with bone deformities or other musculoskeletal pathologies, moment arms estimated from experimental data or generic models are likely to be even less accurate. Although the development of subject-specific models for diverse patient populations will require considerable additional effort, the work presented in this paper represents an important first step.

\section{References}

1. Delp, S. L., Ringwelski, D. A. and Carroll, N. C. (1994) Transfer of the rectus femoris: Effects of transfer site on moment arms about the knee and hip. J Biomech 27, 1201 1211

2. Murray, W. M., Delp, S. L. and Buchanan, T. S. (1995) Variation of muscle moment arms with elbow and forearm position. J Biomech 28, 513-525

3. Arnold, A. S., Komattu, A. V. and Delp, S. L. (1997) Internal rotation gait: a compensatory mechanism to restore abduction capacity decreased by bone deformity. Dev Med Child Neurol 39, 40-44

4. Delp, S. L., Arnold, A. S., Speers, R. A. and Moore, C. A. (1996) Hamstrings and psoas lengths during normal and crouch gait: Implications for muscle-tendon surgery. J Orthop Res 14, 144-151

5. Scott, S. H., Engstrom, C. M. and Loeb, G. E. (1993) Morphometry of human thigh muscles. Determination of fascicle architecture by magnetic resonance imaging. $J$ Anat $182,249-257$

6. Smith, D. K., Berquist, T. H., An, K. N., Rob, R. A. and Chao, E. Y. S. (1989) Validation of three-dimensional reconstructions of knee anatomy: CT vs MR Imaging. Journal of Computer Assisted Tomography 13, 294-301

7. An, K. N., Takahasi, K., Harrigan, T. P. and Chao, E. Y. (1984) Determination of muscle orientations and moment arms. J Biomech Eng 106, 280-282

8. Veldpaus, F. E., Woltring, H. J. and Dortmans, L. J. (1988) A least-squares algorithm for the equiform transformation from spatial marker co-ordinates. J Biomech 21, 45 54

9. Chao, E. Y. and Morrey, B. F. (1978) Three-dimensional rotation of the elbow. J Biomech 11, 57-73

10. Gerbeaux, M., Turpin, E. and Lensel-Corbeil, G. (1996) Musculo-articular modelling of the triceps brachii. J Biomech 29, 171-180

11. London, J. T. (1981) Kinematics of the elbow. J Bone Joint Surg 63-A, 529-535 
12. Shiba, R., Sorbie, C., Siu, D. W., Bryant, J. T., Derek, T., Cooke, V. and Wevers, H. W. (1988) Geometry of the humeroulnar joint. J Orthop Res 6, 897-906

13. Murray, W. M. (1997) The functional capacity of the elbow muscles: Anatomical measurements, computer modeling, and anthropometric scaling. Ph.D. Dissertation Northwestern University, Evanston, IL

14. Delp, S. L. and Loan, J. P. (1995) A graphics-based software system to develop and analyze models of musculoskeletal structures. Comput Biol Med 25, 21-34

15. Delp, S. L. and Zajac, F. E. (1992) Force- and moment-generating capacity of lowerextremity muscles before and after tendon lengthening. Clin Orthop 284, 247-259

16. An, K. N., Hui, F. C., Morrey, B. F., Linscheid, R. L. and Chao, E. Y. (1981) Muscles across the elbow joint: A biomechanical analysis. J Biomech 14, 659-669

17. Amis, A. A., Dowson, D. and Wright, V. (1979) Muscle strengths and musculoskeletal geometry of the upper limb. Eng Med 8, 41-47 